\title{
MODULATION INSTABILITY IN TWO COMPONENT BOSE-EINSTEIN CONDENSATE WITH RELATIVE COMPONENT MOTION
}

\author{
A.P. Ivashin, E.D. Marinenko \\ National Science Center “Kharkov Institute of Physics and Technology”, Kharkiv, Ukraine \\ E-mail: ivashin@kipt.kharkov.ua
}

The development of modulation instability in a spatially homogeneous two-component Bose-Einstein condensate (BEC), in which the interacting components move through each other at a relative speed, is investigated. It is shown that nonlinear dynamics, leading to modulation instability, is determined by both the values of the constant interaction and the relative velocity between the components. The maximum oscillation increment is found and the limits of the existence of modulation instability in the space of wave numbers are determined.

PACS: 03.75.Kk, 03.75.Mn, 03.75.Lm, 03.75.Fi

\section{INTRODUCTION}

When considering the dynamics of systems described by nonlinear equations, as a result of nonlinearity and dispersion interaction processes, weak spatial perturbations in a certain range of wave vectors grow exponentially, creating a chain of localized waves $[1,2]$. In a onecomponent BEC with a negative coupling constant, multiple alternating long-lived domains, which indicated the occurrence of modulation instability (MI) in the system, were experimentally observed [3]. The two component BECs differ significantly from the one component case, where the MI occurs only with a negative interaction between the particles of the condensate. The first MI in two-component BECs was discussed in [4].

When describing the dynamics of BEC at low temperatures, the application of the Gross - Pitaevskii equation [5] has proved effective. Such an approach was used when considering MIs in two-component BECs by many authors [6 - 9]. We applied this approach when describing the dynamics in a two-component BEC with allowance for dissipation [10]. In theoretical papers [11 - 12], within the framework of the GP equation in the case when the stability condition of the system is fulfilled, the occurrence of a MI in a two component BEC with relative component motion was considered. This means that between the constant of intercomponent interaction $g_{12}$ and the constants of intracomponent interaction of particles $g_{1}$ and $g_{2}$ the relation $g_{12}^{2}<g_{1} g_{2}$ is fulfilled [5]. In particular, it was shown the emergence of a critical speed at which stability is broken and a MI arises [11].

The purpose of this article is to clarify the influence of the relative motion of components in a condensate on the development of MI, when the absolute value of the interaction of particles between the components of a condensate can be either greater than the interaction of particles inside the components $\left|g_{12}\right|>g$, or less, $\left|g_{12}\right|<g$. In our case, in order to simplify the calculations, we put $g_{1}=g_{2}$. In both cases, we numerically find the maximum increment and determine the MI instability boundaries in the space of wave numbers.

\section{HYDRODYNAMIC EQUATIONS FOR TWO COMPONENT BEC WITH RELATIVE MOTION OF COMPONENT}

The Gross-Pitaevskii equations for Bose-Einstein condensate for macroscopic wave functions $\psi_{1}(\mathbf{r}, t), \psi_{2}(\mathbf{r}, t)$ have the form [5]

$$
\begin{aligned}
& i \hbar \dot{\psi}_{1}(\mathbf{r}, t)=-\frac{\hbar^{2}}{2 m_{1}} \Delta \psi_{1}(\mathbf{r}, t)+g_{1} \psi_{1}(\mathbf{r}, t)\left|\psi_{1}(\mathbf{r}, t)\right|^{2}+g_{12} \psi_{1}(\mathbf{r}, t)\left|\psi_{2}(\mathbf{r}, t)\right|^{2}-\mu_{1} \psi_{1}(\mathbf{r}, t), \\
& i \hbar \dot{\psi}_{2}(\mathbf{r}, t)=-\frac{\hbar^{2}}{2 m_{2}} \Delta \psi_{2}(\mathbf{r}, t)+g_{2} \psi_{2}(\mathbf{r}, t)\left|\psi_{2}(\mathbf{r}, t)\right|^{2}+g_{21} \psi_{2}(\mathbf{r}, t)\left|\psi_{1}(\mathbf{r}, t)\right|^{2}-\mu_{2} \psi_{2}(\mathbf{r}, t) .
\end{aligned}
$$

We write complex functions $\psi_{1}(\mathbf{r}, t), \psi_{2}(\mathbf{r}, t)$ in the form $\psi_{i}(\mathbf{r}, t)=f_{i}(\mathbf{r}, t) \exp \left[i \varphi_{i}(\mathbf{r}, t)\right]$, where $f_{i}$ and $\varphi_{i}$ are the modules and the phases of functions $\psi_{i}(\mathbf{r}, t)$. Then the density of the number of particles $n_{i}(\mathbf{r}, t)$ and the speed $\mathbf{v}_{i}(\mathbf{r}, t)$ of each component are expressed through the module and phase

$n_{i}(\mathbf{r}, t)=f_{i}^{2}(\mathbf{r}, t), \mathbf{v}_{i}(\mathbf{r}, t)=\frac{\hbar}{m_{i}} \nabla \varphi_{i}(\mathbf{r}, t)$.

In a spatially uniform state, the chemical potentials of the components $\mu_{1}, \mu_{2}$ are associated with equilibrium densities [5]

$$
\mu_{1}=g_{1} n_{10}+g_{12} n_{20}+\frac{m \mathbf{v}_{10}^{2}}{2}, \mu_{2}=g_{2} n_{20}+g_{21} n_{10}+\frac{m \mathbf{v}_{20}^{2}}{2} \text {. }
$$

The index 0 at density and velocity $\left(n_{i 0}, \mathbf{v}_{i 0}\right)$ means that this value refers to its equilibrium value. Let us single out in the equations (1) the real and imaginary parts. Then we have

$$
\begin{aligned}
& \hbar \dot{\varphi}_{1}=\left(\hbar^{2} / 2 m_{1} f_{1}\right)\left(\Delta f_{1}-f_{1}\left(\nabla \varphi_{1}\right)^{2}\right)- \\
& -\left(g_{1} f_{1}^{2}+g_{12} f_{2}^{2}+m \mathbf{v}_{10}^{2} / 2-\mu_{1}\right)-\hbar \mathbf{v}_{10} \nabla \varphi_{1} ; \\
& \hbar \dot{\varphi}_{2}=\left(\hbar^{2} / 2 m_{2} f_{2}\right)\left(\Delta f_{2}-f_{2}\left(\nabla \varphi_{2}\right)^{2}\right)- \\
& -\left(g_{2} f_{2}^{2}+g_{12} f_{1}^{2}+m \mathbf{v}_{20}^{2} / 2-\mu_{2}\right)-\hbar \mathbf{v}_{20} \nabla \varphi_{2} ; \\
& \hbar \dot{f}_{1}=-\left(\hbar^{2} / 2 m_{1}\right) f_{10} \Delta \varphi_{1}-\hbar \mathbf{v}_{10} \nabla f_{1} ; \\
& \hbar \dot{f}_{2}=-\left(\hbar^{2} / 2 m_{2}\right) f_{20} \Delta \varphi_{2}-\hbar \mathbf{v}_{20} \nabla f_{2} .
\end{aligned}
$$

We obtained a system of equations for four unknown quantities $f_{1}, f_{2}, \varphi_{1}, \varphi_{2}$, which depends on nine parameters: on the masses of the two components 
of the condensate $m_{1}$ and $m_{2}$, on three coupling constants $g_{1}, g_{2}, g_{12}$, on two chemical potentials $\mu_{1}$ and $\mu_{2}$ and on two speeds $\mathbf{v}_{10}, \mathbf{v}_{20}$. In accordance with (2), the system of equations (3) can be rewritten in a compact hydrodynamic form

$$
\begin{aligned}
& \dot{n}_{1}+\nabla \cdot\left(n_{1} \mathbf{v}_{1}\right)=0, \\
& \dot{n}_{2}+\nabla \cdot\left(n_{2} \mathbf{v}_{2}\right)=0, \\
& m_{1} \dot{\mathbf{v}}_{1}=-\nabla \tilde{\mu}_{1}, \\
& m_{2} \dot{\mathbf{v}}_{2}=-\nabla \tilde{\mu}_{2},
\end{aligned}
$$

where $\tilde{\mu}_{1}=g_{1} n_{1}+g_{12} n_{2}-\left(\hbar^{2} / 2 m_{1}\right)\left(\Delta \sqrt{n_{1}}\right) / \sqrt{n_{1}}+m_{1} \mathbf{v}_{10} \mathbf{v}_{1}$ and $\tilde{\mu}_{2}=g_{2} n_{2}+g_{12} n_{1}-\left(\hbar^{2} / 2 m_{2}\right)\left(\Delta \sqrt{n_{2}}\right) / \sqrt{n_{2}}+m_{2} \mathbf{v}_{20} \mathbf{v}_{2}-$ are the chemical potentials for the first and second components of the condensate, taking into account the quantum pressure. In the resulting system of hydrodynamic equations for a two-component BEC (4), the first two equations are the continuity equations for each component of the condensate, and the two remaining ones are the Euler equations.

\section{GETTING AND SOLUTION OF A DISPERSION EQUATION}

Let us set in (4) $n_{i}=n_{i 0}+\delta n_{i}$ and $\mathbf{v}_{i}=\mathbf{v}_{i 0}+\delta \mathbf{v}_{i}$, where the densities $n_{i 0}$ and velocities $\mathbf{v}_{i 0}$ correspond to the ground state of the condensate, and $\delta n_{i}$, and $\delta \mathbf{v}_{i}$ are small perturbations of the ground state, depending on the time and spatial coordinate $\delta n_{i}=\delta n_{i}(\mathbf{r}, t)$, $\delta \mathbf{v}_{i}=\delta \mathbf{v}_{i}(\mathbf{r}, t)$. Linearizing the system (4) we get $\delta \dot{n}_{1}+\nabla \cdot\left(n_{10} \delta \mathbf{v}_{1}+\mathbf{v}_{10} \delta n_{1}\right)=0$,

$\delta \dot{n}_{2}+\nabla \cdot\left(n_{20} \delta \mathbf{v}_{2}+\mathbf{v}_{20} \delta n_{2}\right)=0$,

$m_{1} \delta \dot{\mathbf{v}}_{1}=-\nabla \delta \tilde{\mu}_{1}$,

$m_{2} \delta \dot{\mathbf{v}}_{2}=-\nabla \delta \tilde{\mu}_{2}$,

$\delta \tilde{\mu}_{1}=g_{1} \delta n_{1}+g_{12} \delta n_{2}-\left(\hbar^{2} / 2 m_{1}\right) \Delta \delta n_{1} / 2 n_{10}+m_{1} \mathbf{v}_{10} \delta \mathbf{v}_{1}$,

$\delta \tilde{\mu}_{2}=g_{2} \delta n_{2}+g_{12} \delta n_{1}-\left(\hbar^{2} / 2 m_{2}\right) \Delta \delta n_{2} / 2 n_{20}+m_{2} \mathbf{v}_{20} \delta \mathbf{v}_{2}$.

We will seek the solution of the system (5) in the form

$$
\delta n, \delta \mathbf{v} \sim e^{-i(\omega t-\mathbf{k} \cdot \mathbf{r})} .
$$

In this case, the system of equations for $\delta n_{1}, \delta n_{1}, \delta \mathbf{v}_{1}, \delta \mathbf{v}_{2}\left(\varepsilon_{i}^{0}=(\hbar k)^{2} / 2 m_{i}\right)$ takes the form

$-\left(\omega-\mathbf{k} \mathbf{v}_{10}\right) \delta n_{1}+n_{10} \mathbf{k} \delta \mathbf{v}_{1}=0$

$-\left(\omega-\mathbf{k} \mathbf{v}_{20}\right) \delta n_{2}+n_{20} \mathbf{k} \delta \mathbf{v}_{2}=0$,

$\mathbf{k}\left(g_{1}+\varepsilon_{1}^{0} / 2 n_{10}\right) \delta n_{1}+\mathbf{k} g_{12} \delta n_{2}-m_{1}\left(\omega-\mathbf{k} \mathbf{v}_{10}\right) \delta \mathbf{v}_{1}=0$,

$\mathbf{k}\left(g_{2}+\varepsilon_{2}^{0} / 2 n_{20}\right) \delta n_{2}+\mathbf{k} g_{12} \delta n_{1}-m_{2}\left(\omega-\mathbf{k} \mathbf{v}_{20}\right) \delta \mathbf{v}_{2}=0$.

From the obtained system of equations (6) follows the dispersion equation

$$
\begin{aligned}
& (\hbar \omega)^{4}+2(\hbar \omega)^{3}\left\{\hbar \mathbf{k}\left(\mathbf{v}_{10}+\mathbf{v}_{20}\right)\right\}+(\hbar \omega)^{2}\left\{\hbar^{2} k^{2}\left(v_{10}^{2}+4 v_{10} v_{20}+v_{20}^{2}\right)-\left(\varepsilon_{1}^{2}+\varepsilon_{2}^{2}\right)\right\}-2(\hbar \omega)\left\{\left(\hbar \mathbf{k} \mathbf{v}_{10}\right)\left(\hbar \mathbf{k} \mathbf{v}_{20}\right) \hbar \mathbf{k}\left(\mathbf{v}_{10}+\mathbf{v}_{20}\right)-\right. \\
& \left.-\left(\hbar \mathbf{k} \mathbf{v}_{10}\right) \varepsilon_{2}^{2}-\left(\hbar \mathbf{k} \mathbf{v}_{20}\right) \varepsilon_{1}^{2}\right\}+\left(\hbar \mathbf{k} \mathbf{v}_{10}\right)^{2}\left(\hbar \mathbf{k} \mathbf{v}_{20}\right)^{2}-\left(\hbar \mathbf{k} \mathbf{v}_{10}\right)^{2} \varepsilon_{2}^{2}-\left(\hbar \mathbf{k} \mathbf{v}_{20}\right)^{2} \varepsilon_{1}^{2}+\left\{\varepsilon_{1}^{2} \varepsilon_{2}^{2}-4 n_{10} n_{20} g_{12}^{2} \varepsilon_{1}^{0} \varepsilon_{2}^{0}\right\}=0 .
\end{aligned}
$$

Here is the designation $\varepsilon_{i}^{2}=\left(2 g_{i} n_{i 0}+\varepsilon_{i}^{0}\right) \varepsilon_{i}^{0}$. In view of the cumbersome form of the roots of the fourthorder equation obtained, with respect to physical clarity of the solutions, let us make some simplifications. Let us turn into the center of mass system, in which the total momentum of the moving condensates is zero.

Let $m_{1}=m_{2}=m, \quad n_{10}=n_{20}=n$ and $g_{1}=g_{2}=g$ (with $\varepsilon_{1}^{2}=\varepsilon_{2}^{2}=\varepsilon^{2}, \varepsilon_{1}^{0}=\varepsilon_{2}^{0}=\varepsilon^{0}$ ), then the condition of equality to zero of the total pulse will look like $\mathbf{v}_{10}+\mathbf{v}_{20}=0$, and the dispersion equation (7) takes the form

$$
\begin{aligned}
& (\hbar \omega)^{4}-2(\hbar \omega)^{2}\left(\varepsilon^{2}+\hbar^{2} k^{2} v_{R}^{2} / 4\right)+ \\
& +\left(\varepsilon^{2}-\hbar^{2} k^{2} v_{R}^{2} / 4\right)^{2}-4 n^{2} g_{12}^{2}\left(\varepsilon^{0}\right)^{2}=0,
\end{aligned}
$$

where the designation for the relative velocity of the component $v_{R} \equiv\left|\mathbf{v}_{10}-\mathbf{v}_{20}\right|=2\left|\mathbf{v}_{10}\right|$ is entered. The obtained biquadratic equation is easily solved

$$
\left(\hbar \omega^{ \pm}\right)^{2}=\varepsilon^{2}+\hbar^{2} k^{2} v_{R}^{2} / 4 \pm \sqrt{\varepsilon^{2} \hbar^{2} k^{2} v_{R}^{2}+4 n^{2} g_{12}^{2}\left(\varepsilon^{0}\right)^{2}} \text {. }
$$

Thus, in a two-component condensate with components moving relative to each other with speed $v_{R}$, there are two oscillation branches $\omega^{+}$and $\omega^{-}$, that correspond to different signs before the root in (9). In the absence of relative motion of the components of the condensate, the solutions of equation (8) have the form

$\left(\hbar \omega_{0}^{ \pm}\right)^{2}=\varepsilon^{2} \pm 2 n\left|g_{12}\right| \varepsilon^{0}=\varepsilon^{0}\left(\varepsilon^{0}+2 n g \pm 2 n\left|g_{12}\right|\right)$. (10)

In the absence of interaction, we obtain the known spectrum obtained by Bogolyubov for a singlecomponent condensate [5].
In the case of real frequencies, when at a certain ratio between the parameters $g, g_{12}, v_{R}$, the condition $\left(\hbar \omega^{ \pm}\right)^{2}>0$ is fulfilled, the system will be stable and there will be two oscillation branches with frequencies $\omega^{ \pm}$in it. Let us clarify the question when the stability of a system with respect to small perturbations is broken and a modulation instability arises [6].

\section{CONDITIONS OF MI IN THE BEC WITH RELATIVE MOVEMENT COMPONENT}

The expressions (9) and (10) can be written in a dimensionless form. To do this, we introduce the notation

$$
E^{ \pm} \equiv\left(\hbar \omega^{ \pm}\right) /(n g), \tilde{v}_{R} \equiv v_{R} / c,
$$

$$
\gamma \equiv\left|g_{12}\right| / g E_{0}^{ \pm} \equiv\left(\hbar \omega_{0}^{ \pm}\right) /(n g), \tilde{k}^{2} \equiv \hbar^{2} k^{2} /(2 m n g),
$$

and $c=n g / m$ is the speed of sound in the condensate. As a result, we obtain an expression for the dependence of the dimensionless oscillation energy $E$ on the dimensionless wave vector $\tilde{k}$ and the dimensionless velocity $\tilde{v}_{R}$

$$
\left(E^{ \pm}\right)^{2}=\tilde{k}^{2}\left(\tilde{k}^{2}+2+\frac{\tilde{v}_{R}^{2}}{2} \pm 2 \sqrt{\left.\frac{\tilde{v}_{R}^{2}}{2}\left(\tilde{k}^{2}+2\right)+\gamma^{2}\right)} .\right.
$$

And for the occasion $\tilde{v}_{R}=0$

$$
\left(E_{0}^{ \pm}\right)^{2}=\tilde{k}^{2}\left(\tilde{k}^{2}+2(1 \pm \gamma)\right) .
$$

Modulation instability in the system arises when expressions (11) and (12) for $\left(E^{ \pm}\right)^{2}$ and $\left(E_{0}^{ \pm}\right)^{2}$ are negative 


$$
\begin{gathered}
\tilde{k}^{2}+2+\frac{\tilde{v}_{R}^{2}}{2} \pm 2 \sqrt{\frac{\tilde{v}_{R}^{2}}{2}\left(\tilde{k}^{2}+2\right)+\gamma^{2}}<0, \\
\tilde{k}^{2}+2(1 \pm \gamma)<0 .
\end{gathered}
$$

From the expression (11) and (12) it can be seen that this is possible only for $\left(E^{-}\right)^{2}$ and $\left(E_{0}^{-}\right)^{2}$ (the second oscillation branch with frequencies $\omega^{-}$and $\omega_{0}^{-}$, respectively), since the expressions for $\left(E^{+}\right)^{2}$ and $\left(E_{0}^{+}\right)^{2}$ are always positive. A negative value $\left(E^{-}\right)^{2}$ indicates that the frequency $\omega^{-}$is a purely imaginary quantity, and it can be represented as $\omega^{-}=i G$. Here $G$ (the absolute value $\left.\omega^{-}\right)$, is an oscillation increment. Let us introduce the value $\tilde{G} \equiv(\hbar G) /(n g)$, corresponding to the dimensionless increment of oscillations in the event of MI. We have from (11)

$$
\begin{aligned}
& E^{-}=\left(\hbar \omega^{-}\right) /(n g)=i(\hbar G) /(n g)=i \tilde{G}, \\
& \tilde{G}^{2}=\tilde{k}^{2}\left(2 \sqrt{\frac{\tilde{v}_{R}^{2}}{2}\left(\tilde{k}^{2}+2\right)+\gamma^{2}}-\left(\tilde{k}^{2}+2+\frac{\tilde{v}_{R}^{2}}{2}\right)\right) .
\end{aligned}
$$

In the absence of relative motion the components of the condensate MI exist in the region of wave vectors $0<\tilde{k}^{2}<2(\gamma-1)(14)$. It follows that the modulation instability in this case exists only for $\gamma>1$, i.e. when the absolute value of the interparticle interaction between the components of the condensate is greater than the intracomponent interaction of particles $\left|g_{12}\right|>g$. The boundary value of the wave vector for the existence of MI according to (14)

$$
\tilde{k}_{B}^{2}=2(\gamma-1) .
$$

Or in dimensional units [8]

$$
k_{B}^{2}=\frac{4 m n g}{\hbar^{2}}\left(\frac{\left|g_{12}\right|}{g}-1\right) .
$$

When $\tilde{v}_{R}=0$, the mode of oscillation, at which the increment of the MI will be maximum, is determined by the condition [6]

$$
\partial\left(E_{0}^{-}\right)^{2} / \partial \tilde{k}^{2}=0
$$

According to (12) we have $\left(E_{0}^{-}\right)^{2}=\tilde{k}^{2}\left(\tilde{k}^{2}+2(1-\gamma)\right)$. Then the maximum wave vector $k_{\max }$, for which there is a maximum oscillation increment $G_{\max }$, is

$$
\tilde{k}_{\max }^{2}=\gamma-1 \text {. }
$$

Or in dimensional units [8]

$$
k_{\max }=\sqrt{\frac{2 m n g}{\hbar^{2}}\left(\frac{\left|g_{12}\right|}{g}-1\right)} .
$$

Substituting $\tilde{k}^{2}=(\tilde{k})_{\max }^{2}$ in expression (15) we find the maximum increment $\tilde{G}_{\max }$

$$
\tilde{G}_{\max }=\sqrt{(\tilde{k})_{\max }^{2}\left(2(\gamma-1)-(\tilde{k})_{\max }^{2}\right)}=(\tilde{k})_{\max }^{2} .
$$

And, accordingly, the increment $G_{\max }$ is equal (see [6])

$$
G_{\max }=\hbar(k)_{\max }^{2} / 2 m=(n g / \hbar)(\gamma-1) .
$$

We now take into account the motion of the components of the condensate relative to each other. According to (11)

$$
\left(E^{-}\right)^{2}=\tilde{k}^{2}\left(\tilde{k}^{2}+2+\frac{\tilde{v}_{R}^{2}}{2} 2 \sqrt{\frac{\tilde{v}_{R}^{2}}{2}\left(\tilde{k}^{2}+2\right)+\gamma^{2}}\right) .
$$

This expression becomes negative when

$$
\left|\tilde{k}^{2}+2-\frac{\tilde{v}_{R}^{2}}{2}\right|<2 \gamma \text {. }
$$

Thus, MI when $v_{R} \neq 0$ exists for wave vectors

$$
-2(\gamma+1)+\frac{\tilde{v}_{R}^{2}}{2}<\tilde{k}^{2}<2(\gamma-1)+\frac{\tilde{v}_{R}^{2}}{2} .
$$

This inequality determines the zone of MI for different values of speed $\tilde{v}_{R}$ and parameter $\gamma$. Expression (17) is true for cases $\gamma>0$ when, i.e. when intercomponent interaction can be both more and less interaction between particles inside the condensate component. This is a significant difference from [8,11], where only the case $\gamma<1$ was considered. The boundary values of the wave vectors $\tilde{k}_{B 1}^{2}$ and $\tilde{k}_{B 2}^{2}$ are equal

$$
\tilde{k}_{B 1}^{2}=2(\gamma-1)+\frac{\tilde{v}_{R}^{2}}{2}, \tilde{k}_{B 2}^{2}=-2(\gamma+1)+\frac{\tilde{v}_{R}^{2}}{2} .
$$

From (17) it follows that in the case when $\gamma<1$, for velocities $\tilde{v}_{R}^{2}<4(1-\gamma)$ MI is absent for all values $\tilde{k}^{2}$. Thus, in a two-component BEC with relative motion of components, there is a critical speed equal to [11]

$$
\left(\tilde{v}_{R}^{2}\right)_{c r}=4(1-\gamma) \text {. }
$$

The quantity $\tilde{G}$ from (15) is a function of two variables: $\tilde{k}^{2}$ and $\tilde{v}_{R}^{2}$. We find numerically the maximum value of this function (maximum oscillation increment) for various values of the parameters $\gamma$. The results are shown in the table. (For comparison, the table shows the corresponding increment values for the case $v_{R}=0$ ).

\section{Dependence of the maximum increment $\tilde{G}_{\max }$}

on the parameter $\gamma$

\begin{tabular}{|c|c|c|}
\hline$\gamma$ & $\tilde{G}_{\max }\left(v_{R} \neq 0\right)$ & $\tilde{G}_{\max }\left(v_{R}=0\right)$ \\
\hline 0.3 & 0.025 & 0 \\
\hline 0.707 & 0.333 & 0 \\
\hline 1 & 0.75 & 0 \\
\hline 2 & 3.385 & 1 \\
\hline 3 & 6.75 & 2 \\
\hline
\end{tabular}

The Table shows that the increment values $\tilde{G}_{\max }$ in the first case $\left(v_{R} \neq 0\right)$ are larger than in the absence of relative movement of the condensate components. Thus, the relative movement of the components of the condensate leads to a more rapid development of MI.

Consider the long-wave approximation. Suppose that the relations between the interaction parameters $g, g_{12}$ and relative velocity $v_{R}$ are such that the condition $\left(\hbar \omega^{ \pm}\right)^{2}>0$ is satisfied, i.e. frequencies are real. When $\lambda>>\xi$ (where $\xi=\hbar / \sqrt{2 m n g}$ is the coherence length in BEC), we have $\tilde{k}^{2}<<1$. Let's pass to dimensional variables. According to (9) oscillation spectrum

$$
\left(\omega^{ \pm}\right)^{2}=\frac{1}{\hbar^{2}}\left(\varepsilon^{2}+\hbar^{2} k^{2} v_{R}^{2} / 4 \pm \sqrt{\varepsilon^{2} \hbar^{2} k^{2} v_{R}^{2}+4 n^{2} g_{12}^{2}\left(\varepsilon^{0}\right)^{2}}\right)
$$

in the long-wave region will be 


$$
\omega^{ \pm}=k \sqrt{c^{2}+v_{R}^{2} / 4 \pm \sqrt{c^{2} v_{R}^{2}+\left(n g_{12} / m\right)^{2}}} .
$$

From this it follows that the dependence of the oscillation frequency on the wave vector in the long-wave limit has a qualitatively the same form as in the singlecomponent Bose condensate moving with speed $v_{0}$

$$
\omega=k v_{0} \pm k c \text {. }
$$

\section{CONCLUSIONS}

In the absence of relative motion of the components in the BEC, modulation instability exists only when $g_{12}>g(\gamma>1)$, i.e. when the interaction forces between the atoms inside the components are less than the intercomponent interaction forces. When the system has movement of components relative to each other $\left(v_{R} \neq 0\right)$, the situation changes. In this case, the condition $\gamma>1$ is no longer decisive for the occurrence of MI. The occurrence of MI in a moving two-component BEC is now determined by the ratio between the magnitudes of the constant interactions between particles and the relative velocity between the components of the condensate. Moreover, the value $\gamma$ can be either greater or less than 1. Note that the development of MI is not affected by the sign of the interaction between the components.

It is shown that in the linear approximation with respect to density and velocity perturbations in the BEC, the presence of relative motion of the condensate components changes the boundary values of the wave vectors at which the MI exists, expanding the range of the MI existence. The presence of relative motion increases the maximum increment of the instability of the MI. It is also shown that for stable oscillations in the longwavelength limit, the oscillation frequency linearly depends on the wave vector, as in the case of onecomponent condensate.

\section{REFERENCES}

1. G.P. Agraval. Modulation Instability Induced by Cross-Phase Modulation // Phys. Rev. Lett. 1987, v. 59 , p. $880-883$.
2. E.A. Donley, N.R. Claussen, S.L. Cornish, J.L. Roberts, E.A. Cornell. Dynamics of collapsing and exploding Bose-Einstein condensates // Nature. 2001, v. 412, p. 295-299.

3. H.J. Miesner, D.M. Stamper-Kurn, J. Stenger, S. Inouye, A.P. Chikkatur, and W. Ketterle. Observation of Metastable States in Spinor Bose-Einstein Condensates // Phys. Rev. Lett. 1999, v. 82, p. 2228-2231.

4. E.W. Goldstein and P. Meystre. Quasiparticle instabilities in multicomponent atomic condensates // Phys. Rev. A. 1997, v. 55, p. 2935-2940.

5. C.J. Pethick, H. Smith. Bose-Einstein Condensation in Dilute Gases // 2nd edn. Cambridge: Cambridge University Press, 2008, 569 p.

6. P. Ao, S.T. Chui. Two Stages in Evolution of Binary Alcali BEC Mixtures towards Phase Segregation // Phys. Rev. A. 1998, v. 58, p. 4836-4840, arXiv:condmat/0003185. 2000, 17 p.

7. L.D. Carr, J. Brand. Spontaneous Soliton Formation and Modulational Instability in Bose-Einstein Condensates // Phys. Rev. Lett. 2004, v. 92, p. 040401.

8. K. Kasamatsu, M. Tsubota. Modulation instability and solitary-wave formation in two-component Bose-Einstein condensate // Phys. Rev. A. 2006, v. 74, p. 013617.

9. S. Ronen, J.L. Bohn, L.E. Halmo and M. Edwards. Dynamical pattern formation during growth of a dual-species Bose-Einstein condensate // Phys. Rev. A. 2008, v. 78, p. 053613.

10. A.P. Ivashin, E.D. Marinenko. Modulation instability in two component Bose-Einstein condensate with dissipation // East. Eur. J. Phys. 2019, v. 1, p. 65-74.

11. C.K. Law, C.M. Chan, P.T. Leung, and M.-C. Chu. Critical velocity in a binary mixture of moving Bose condensates // Phys. Rev. A. 2001, v. 63, p. 063612 $(1-4)$.

Article received 31.05.2019

\section{МОДУЛЯЦИОННАЯ НЕУСТОЙЧИВОСТЬ В ДВУХКОМПОНЕНТНОМ БОЗЕ-ЭЙНШТЕЙНОВСКОМ КОНДЕНСАТЕ ПРИ ОТНОСИТЕЛЬНОМ ДВИЖЕНИИ КОМПОНЕНТ}

\section{А.П. Ивашин, Е.Д. Мариненко}

Исследуется развитие модуляционной неустойчивости в пространственно-однородном двухкомпонентном бозе-эйнштейновском конденсате (БЭК), в котором взаимодействующие компоненты движутся друг относительно друга со скоростью $v_{R}$. Показано, что нелинейная динамика, приводящая к модуляционной неустойчивости, определяется как величинами постоянных взаимодействия, так и относительной скоростью между компонентами. Найден максимальный инкремент колебаний и определены границы существования модуляционной неустойчивости в пространстве волновых чисел.

\section{МОДУЛЯЦІЙНА НЕСТІЙКІСТЬ У ДВОКОМПОНЕНТНОМУ БОЗЕ-ЕЙНШТЕЙНІВСЬКОМУ КОНДЕНСАТІ ПРИ ВІДНОСНОМУ РУХОВІ КОМПОНЕНТ}

\section{А.П. Івашин, О.Д. Маріненко}

Досліджується розвиток модуляційної нестійкості в просторово-однорідному двокомпонентному бозеейнштейнівському конденсаті (БЕК), в якому взаємодіючі компоненти рухаються один відносно одного зі швидкістю $v_{R}$. Показано, що нелінійна динаміка, яка веде до модуляційної нестійкості, визначається як величинами постійних взаємодій, так і відносною швидкістю між компонентами. Знайдено максимальний інкремент коливань і визначено межі існування модуляційної нестійкості в просторі хвильових чисел. 